\title{
Tradução e Adaptação do Conteúdo: A-19, Scale of Children's Attitudes, de Grims e Guitar (1977)
}

\section{Translation and Adaptation of the Content: A-19, Scale of Children's Attitudes, by Grims \& Guitar (1977)}

\author{
Catarina Fitas*, Nádia Marques*, Vanessa Sousa*, Elsa Soares** \\ * Escola Superior de Saúde de Leiria, ** Instituto Politécnico de Leiria
}

\begin{abstract}
Resumen
Os sentimentos e atitudes na gaguez podem originar na criança angústia e frustração. Atendendo à importância de se avaliarem estas dimensões e, na ausência de instrumentos validados para o português europeu, considerou-se importante proceder à tradução e adaptação transcultural da Escala A-19. Este artigo descreve as primeiras três fases, qualitativas, desse processo. Realizaram-se duas traduções independentes e síntese das mesmas. Para verificar inconsistências ou erros conceptuais efetuaram-se duas retrotraduções independentes, concluindo-se que a tradução realizada foi consistente com o instrumento original. A presente investigação permitiu obter o conteúdo da "A-19, Scale of Children's Attitudes traduzido para Portugués Europeu.

Palabras clave: gaguez, avaliação, instrumento, escala A-19, crianças.
\end{abstract}

\begin{abstract}
The feelings and attitudes in stuttering can produce in the child distress and frustration. Given the importance of assessing these dimensions and, in the absence of validated instruments for European Portuguese, it was considered important to proceed with the cross-cultural adaptation of Scale A-19. This article describes the first three qualitative phases of this process. Two independent translations and synthesis of the same were made. In order to verify inconsistencies or conceptual errors, two independent backtranslations were carried out, concluding that the translation was consistent with the original instrument

The present investigation allowed to obtain the content of the A-19, Scale of Children's Attitudes translated to european portuguese.

Keywords: stuttering, evaluation, instrument, scale A-19, children.
\end{abstract}

A gaguez pode definir-se como uma perturbação na fluência normal e padronização temporal da fala que persiste ao longo do tempo. Caracteriza-se pela ocorrência de comportamentos primários (repetições, prolongamentos e bloqueios), secundários (comportamentos de escape e de evitamento) aos quais se podem associar atitudes e sentimentos negativos (Guitar,
2014; Manning, 2010; Shipley \& McAfee, 2015; American Psychiatric Association, 2014).

Existe uma relação entre a gaguez e os sentimentos $e$ atitudes. A gaguez pode causar sentimentos de grande dor, angústia e frustração (Guttormsen, Kefalianos, \& Naess, 2015; Guitar, 2014; Manning, 2010); a pessoa que gagueja pode vir a ser alvo de pena, correções, gozo, ridicularização, castigo, evitamento, isolamento e desprezo devido à perturbação (Shipley \& McAfee, 2015; Guitar, 2014; Manning, 2010). Tal torna-se importante desconstruir na intervenção (Guitar, 2014; Manning, 2010).

Estudos demonstram que o temperamento das crianças que gaguejam pode influenciar a reação à gaguez pelo que é possível identificar crianças que têm uma menor capacidade de regular as emoções, contribuindo para o agravamento da perturbação e risco aumentado de gaguejar (Jones, Choi, Conture, \& Walden, 2014). Em paralelo, sabe-se que crianças que gaguejam apresentam mais atitudes negativas, comparativamente aos seus pares, na comunicação; tal é considerado por Guttormsen, Kefalianos \& Naess (2015), como possível influência no desenvolvimento ou persistência da gaguez.

Atendendo a esta relação e dado que não existe, em Portugal, nenhum instrumento validado, neste âmbito, que possam ser utilizado pelos terapeutas da fala. Na primeira fase da investigação foram delineados dois objetivos de investigação: 1) tradução, e 2) adaptação do conteúdo para o português europeu, da A-19, Scale of Children's Atittudes, de Grims \& Guitar (1977).

Este instrumento destina-se a ser aplicado a crianças que frequentam o ensino pré-escolar e/ou básico, e tem como finalidade aferir como estas se sentem em relação à sua própria gaguez, avaliando as suas atitudes sobre a fala (Guitar, 2014; Manning, 2010). 


\section{Métodos}

\section{Instrumentos}

A A-19, Scale of Children's Atittudes, de Grims e Guitar (1977) caracteriza-se por 19 questões fechadas que avaliam as atitudes da criança sobre a sua gaguez em diversos contextos, familiar e escolar, e interlocutores associados. A escala é constituída por uma introdução e um questionário. $\mathrm{Na}$ introdução constam as instruções de aplicação, ilustradas com um exemplo prático de um possível diálogo a estabelecer-se entre o profissional e a criança; posteriormente, é apresentado um quadro com a cotação disponível para cada uma das respostas, sendo que a atribuição de 1 ou 0 pontos está dependente da especificidade da questão. Quanto mais alto for o resultado obtido, maior a probabilidade de a criança ter atitudes negativas relativamente à sua comunicação

\section{Materias e Procedimentos}

A tradução e adaptação do conteúdo deste instrumento recorreu a uma metodologia de investigação qualitativa. Os instrumentos de medição quando se destinam a ser utilizados noutras culturas devem ser sujeitos a tradução e adaptação cultural por forma a manterem a sua validade conceptual (Beaton, Guillemin, \& Ferraz, 2000). Atendendo a tal, a presente investigação foi suportada nas linhas orientadoras de Beaton, Guillemin, \& Ferraz (2000) que recomendam que o processo de adaptação transcultural decorra em seis fases: fase 1 - tradução inicial; fase 2 - síntese das traduções; fase 3 retrotradução; fase 4 - comité de especialistas; fase 5 teste da versão pré-final e 6 - submissão da documentação aos seus autores ou comité para aprovação do processo de adaptação.

Inicialmente, e por forma a garantir o respeito pelos direitos de autor (Wild, Grove, Martin, Eremenco, McElroy, Verjee-Lorenz \& Erikson, 2005) contactou-se um dos autores do instrumento original, Barry Guitar, por forma a obter-se a sua permissão para a tradução, adaptação e validação da escala Scale of Children's Atittudes, A-19. Após permissão concedida iniciou-se o processo de adaptação transcultural, nomeadamente, a fase 1.

A escolha das tradutoras foi orientada pelos requisitos inerentes à fase 1 da metodologia proposta por Beaton, Guillemin, \& Ferraz (2000). Após convite colaboraram duas tradutoras independentes, fluentes na língua inglesa. A primeira, licenciada em Filologia Germânica (TT1) e a segunda, em Línguas e Literaturas Modernas - Variante Inglês/Alemão, bilingüe (TT2). Para a obtenção destas informações foi solicitado pessoalmente o preenchimento de um questionário sociodemográfico a cada uma das profissionais. Foi também assegurado o direito à confidencialidade.

Após a leitura minuciosa de ambas as traduções, e, considerando o objetivo desta fase da investigação, procedeu-se à definição de categorias de análise. Considerou-se relevante definirem-se como categorias de análise as palavras/expressões/ frases que não reuniram consenso entre traduções. Sublinharam-se as mesmas e procedeu-se à elaboração de um documento com cada uma destas
A segunda fase, que respeita à síntese das traduções (Beaton, Guillemin \& Ferraz, 2000), caracterizou-se pela reunião das tradutoras e investigadoras no sentido de obter-se uma versão consensual do instrumento. Estas constituiram-se como um dos grupos de enfoque da presente investigação dado que permite explorar as perceções, experiências e significados de cada um dos seus membros em relação ao objeto de investigação (Coutinho, 2014).

Neste grupo de enfoque comparam-se as duas versões das traduções e alcançou-se um consenso sobre as expressões em análise.

A terceira fase, retrotradução, corresponde, de acordo com Beaton, Guillemin \& Ferraz (2000), à tradução para a língua de origem da versão final obtida na fase anterior. Esta contou com a colaboração de duas docentes, ambas do Ensino Superior. A mesma foi realizada por uma Terapeuta da Fala, doutorada em Estudos da Criança, especializada em Educação Especial (RTT1), e por uma Terapeuta Ocupacional, mestre em Ciências de Neuro Reabilitação, bilingue (RTT2). Até à realização da retrotradução nenhuma das profissionais havia tido contacto prévio com o instrumento original.

Obteve-se a primeira versão das retrotraduções, RTT1 e RTT2, a partir das quais se elaborou um relatório comparativo. Para a conclusão desta fase, definiram-se como elementos do grupo de enfoque as duas retrotradutoras. Pretendia-se aferir se o conteúdo da versão do idioma original estava preservado, alcançandose, também, uma versão final de consenso satisfatória.

\section{Resultados}

Os resultados decorrentes do processo de tradução aferiram 27 divergências entre as versões traduzidas.

Aquando a fase 1 verificou-se que a TT1 realizou, comparativamente a TT2, uma tradução com um léxico e estrutura sintática mais formal. A TT2, por seu turno, apresentou uma tradução mais literal do conteúdo da língua de origem recorrendo a um léxico mais simples.

Relativamente ao instrumento, a maioria dos itens foi traduzido de forma consensual. Nas expressões/ palavras/ frases divergentes não se destacou nenhum item de difícil discussão pelo que os consensos alcançados não envolveram oposição entre intervenientes.

Nos itens correspondentes às instruções e a questões da escala, as tradutoras tiveram em consideração a complexidade lexical e morfossintática dos enunciados dada a faixa etária da população-alvo.

$\mathrm{Na}$ palavra administration para além da equivalência semântica, realizou-se a equivalência conceptual; considerou-se que a palavra «administração» não seria a tradução mais adequada, no português europeu. $\mathrm{Na}$ expressão keep your mouth shut procedeu-se à equivalência idiomática; na língua portuguesa, não é usado o coloquialismo «manter a boca fechada».

A partir dos vários consensos, obteve-se a primeira versão da escala (T3) que, posteriormente, foi objeto de retrotradução por duas tradutoras.

De um modo geral, as retrotraduções foram congruentes entre si e com o instrumento original. 
Relativamente às palavras, termos e frases retrotraduzidas, a RTT2 igualou a tradução ao instrumento original 18 das 79 divergências. A RTT1, por seu turno, igualou-a em 1 item.

Verificaram-se algumas divergências entre a versão original na língua inglesa e as retrotraduções elaboradas pelas retrotradutoras; estas ocorreram em 24 palavras, frases e/ou expressões e relacionaram-se com a estrutura sintática, constituintes frásicos e variação verbal utilizados. Em alguns casos omitiram-se palavras para reduzir a redundância (repetição de palavras) no texto escrito. Ainda assim, não se verificou prejuízo do conteúdo semântico.

Em 26 das palavras, frases e/ou expressões substituíram-se os termos originais por equivalentes.

Os itens physically, quickly before he or she is ready, you feel, grade stutterers, best to keep your mouth shut $\mathrm{e}$ classmates foram sujeitos a discussão.

Foi consensual, entre todos os intervenientes, que a palavra physically e expressão best to keep your mouth shut se reportavam a expressões idiomáticas que deveriam ser objeto de alteração.

Nas palavras e expressões physically, quickly before he or she is ready e you feel foi sugerida a não inclusão da expressão por não fazer sentido no português europeu. $\mathrm{Na}$ expressão grade stutterers, a versão original contempla no seu significado o conceito de estudante, no entanto, na retrotradução, ambas as retrotradutoras procederam a uma tradução literal, nomeadamente grade stutterers students.

No palavra classmates considerou-se que a melhor retrotradução a adotar seria 12. (...) colleagues dado que, na língua portuguesa é mais usual usar-se a palavra «colegas» quando se fala em colegas da escola. Na língua inglesa esta expressão já não faz sentido, pois é usada associada a contextos de trabalho.

As principais divergências encontradas entre as retrotraduções relacionaram-se com o facto das retrotradutoras terem traduzido, de forma literal, as palavras, expressões e/ou frases da versão de consenso T3 realizada pelas tradutoras.

\section{Discussão}

A metodologia qualitativa para a tradução e adaptação de conteúdo de instrumentos de avaliação foi fundamental para o enquadramento da escala original, tendo em conta as especificidades da cultura portuguesa.

$\mathrm{Na}$ primeira fase - tradução - são necessárias pelo menos duas traduções do instrumento original para o idioma-alvo, para que, mediante comparação se analisem discrepâncias ou ambiguidades entre as palavras. (Beaton, Guillemin, \& Ferraz, 2000; Wild, et al., 2005; Ferreira, Neves, Campana, \& Tavares, 2014). As tradutoras devem ser naturais do país a que se destina a tradução, e devem ter domínio semântico, conceptual e cultural do idioma do instrumento original (Beaton, Guillemin, \& Ferraz, 2000; Ferreira, Neves, Campana, \& Tavares, 2014).

No âmbito desta investigação, muito embora uma das tradutoras não fosse bilingue, nem nenhuma apresentasse domínio conceptual da escala, ambas possuíam o domínio semântico e cultural do instrumento de origem. Tal permitiu obterem-se traduções que Beaton, Guillemin, \& Ferraz (2000) caracterizam como sendo a linguagem utilizada pela população em geral. Ainda assim, Swaine-Verdier, Doward, Hagell, Thorsen, \& McKenna (2004) referem que, dada a necessidade de manter-se uma linguagem acessível à população-alvo aquando a tradução do instrumento, devem recrutar-se tradutores que contactem com públicos diversificados nomeadamente professores.

Aquando a fase 1 verificou-se que a TT1 realizou, comparativamente a TT2, uma tradução mais formal. Manteve-se esta versão da tradução apenas nas instruções da escala, dado que estas se dirigem a profissionais de saúde. Pensa-se que tal se relevou a opção metodológica adequada. Segundo Swaine-Verdier, Doward, Hagell, Thorsen, \& McKenna (2004), quando as traduções utilizam um estilo demasiado académico tal poderá levar a traduções inapropriadas à população-alvo. Estes autores salientam ainda que os questionários/ inquéritos destinam-se a ser aplicados à população em geral e não a profissionais de saúde, corroborando, assim, a posição de Beaton, Guillemin, \& Ferraz (2000) que defendem que estes instrumentos devem ser de fácil compreensão.

Para a conclusão da fase 2, síntese das traduções (TT1 e TT2), verificaram-se que as divergências corresponderam a equivalências entre palavras/frases que podiam ser interpretadas de forma similar entre as duas culturas. O consenso entre tradutoras foi unânime respeitando as recomendações de Beaton, Guillemin, \& Ferraz (2000).

A consecução da fase 3 pressupõe que os retrotradutores sejam nativos do país do instrumento original e não tenham contacto prévio com o instrumento original e área em estudo (Ferreira, Neves, Campana, \& Tavares, 2014). Na investigação levada a cabo, nenhuma das retrotradutoras tinham como língua materna a língua inglesa; não obstante, a RTT2 esteve, durante 10 anos, no país de origem do instrumento considerando-se, por isso, bilingue. A par de tal, a RTT1 apresentava um certificado de dominio da língua inglesa correspondente ao nível C1 e tinha conhecimento da área em estudo; no entanto, tal não enviesou os objetivos desta etapa metodológica.

A etapa de retrotradução afere se a versão traduzida reflete os conteúdos da versão original, assegurando a consistência da tradução (Ferreira, Neves, Campana, \& Tavares, 2014).

As retrotraduções foram comparadas entre si e verificou-se um grande número de divergências. Estas reportavam-se, contudo, a palavras do mesmo campo semântico e a alterações da estrutura morfossintática de frases que não comprometiam o conteúdo.

Da análise concluiu-se que a tradução realizada pelas tradutoras independentes foi consistente com o instrumento original (nas instruções e na escala). Verificou-se, na maioria dos casos, uma correta tradução literal e conceptual dos conceitos, conforme defendido por Wild, et al., 2005).

Swaine-Verdier, Doward, Hagell, Thorsen, \& McKenna (2004) referem que uma tradução com qualidade pode ser aferida quando a retrotradução não é igual ao instrumento de origem. No estudo levado a cabo 
verificou-se, num universo de 79 itens traduzidos de forma diferente, entre retrotradutoras, que apenas $24 \%$ correspondiam a traduções exatamente iguais à tradução original.

As retrotradutoras referiram, neste contexto, que algumas palavras retraduzidas embora não fizessem sentido na língua inglesa, eram a equivalência mais adequada ao português europeu. Consideraram ainda, nos casos em que foi realizada uma retrotradução literal, que esta poderia não ser a mais adequada ao idioma da línguaalvo, o que corrobora a hipótese aventada pelos autores supracitados.

Constituíram-se limitações da investigação a impossibilidade, até à data, de consulta do estudo original que deu lugar à construção da Escala A-19; o facto de a escala ter sido elaborada na década de 70, o que obrigou à proposta de realização de adaptações congruentes com o contexto sociocultural atual. Relativamente à metodologia, teria sido importante que a língua materna das tradutoras independentes fosse a língua do instrumento; a par de tal, uma destas deveria ter conhecimentos na área a que se reporta o instrumento por forma a fornecer uma perspetiva mais clínica e confiável da tradução. No que tange à retrotradução seria relevante que as duas retrotradutoras fossem bilingues, ainda assim, cumpriu-se o requisito de que nenhuma destas conhecia a escala.

Concluiu-se que os objetivos da presente investigação foram cumpridos. Dada a influência dos sentimentos e atitudes na gaguez revela-se importante dar continuidade a esta investigação. A carência de instrumentos validados, para a população portuguesa, neste âmbito, torna necessário investir-se na construção ou validação de baterias de avaliação. Assim, pretende-se, de futuro, proceder-se à validação transcultural instrumento.

\section{Referências}

American Psychiatric Association. (2014). Manual de Diagnóstico e Estatística das Perturbações Mentais (5. ${ }^{a}$ Edição). Lisboa: Climepsi Editores.

Beaton, D. E., Guillemin, F., \& Ferraz, M. B. (2000). Guidelines for the process of cross-cultural adaptation of self-report measures. Spine, 3186-3191. doi:http://dx.doi.org/10.1097/00007632-20001215000014.

Coutinho, C. P. (2014). Metodologia de Investigação em Ciências Sociais e Humanas: Teoria e Prática (2 ed.). Coimbra: Almedina.

Ferreira, L., Neves, A., Campana, M., \& Tavares, M. (2014). Guia da AAOS/IWH: sugestões para a adaptação transcultural de escalas. Avaliação Psicológica, 13(3), 457-461. Obtido de http://pepsic.bvsalud.org/pdf/avp/v13n3/v13n3a18.pdf

Guitar, B. (2014). Stuttering: An Integrated Approach to Its Nature and Treatment. Fourth Edition (4 ed.). Philadelphia , United States: Wolters Kluwer Health/Lippincott Williams \& Wilkins .

Guttormsen, L. S., Kefalianos, E., \& Naess, K.-A. B. (2015). Communication attitudes in children who stutter: A meta-analytic review. Journal of Fluency
Disorders, $1-14$. doi:http://dx.doi.org/10.1016/j.jfludis.2015.08.001

Jones, R., Choi, D., Conture, E., \& Walden, T. (2014). Temperament, Emotion and Childhood Stuttering. Seminars in Speech and Language, 114-131. doi:https://dx.doi.org/10.1055\%2Fs-0034-1371755

Manning, W. H. (2010). Clinical Decision Making in Fluency Disorders. (3 ed.). USA: Cengage Learning.

Shipley, G. K., \& McAfee, G. J. (2015). Assessment in Speech-Language Pathology: A Resource Manual, Fifth Edition (2 ed.). Boston: Cengage Learning.

Silva, I. S., Veloso, A. L., \& Keating, J. B. (2014). Focus group: Considerações teóricas e metodológicas. Revista Lusófona de Educação, 175-190. Obtido de http://revistas.ulusofona.pt/index.php/rleducacao/articl e/view/4703

Swaine-Verdier, A., Doward, L. C., Hagell, P., Thorsen, H., \& McKenna, S. P. (2004). Adapting Quality of Life Instruments. Value in Health, S27-S30. doi:http://dx.doi.org/10.1111/j.15244733.2004.7s107.x

Wild, D., Grove, A., Martin, M., Eremenco, S., McElroy, S., Verjee-Lorenz, A., \& Erikson, P. (2005). Principles of Good Practice for the Translation and Cultural Adaptation Process for Patient-Reported Outcomes (PRO) Measures: Report of the ISPOR Task Force for Translation and Cultural Adaptation. Value in Health, 8, 94-104. doi:http://dx.doi.org/10.1111/j.15244733.2005.04054.x 